\title{
La naturaleza habla, las ciudades gritan
}

Mauricio Muñoz Escalante

Universidad Antonio Nariño

munoz.mauricio@gmail.com

Los abusos contra el ambiente son noticia de todos los días, sin embargo, no siempre es claro cómo extraer de los hechos cotidianos la correspondencia entre la construcción de la ciudad y la protección de la naturaleza. Por ejemplo: si se denuncia que ingresaron maquinaria pesada por la cuenca del río Gaira, dentro de la reserva natural de aves El Dorado, para abrir vías en un proyecto de casas de descanso en la Sierra Nevada de Santa Marta, tal como denunció la Fundación ProAves de Colombia (2015), la relación es incontestable. En todas las fases de la empresa-factibilidad, esquema básico, anteproyecto, construcción-, es fácil determinar la responsabilidad de cada uno de los involucrados con el ambiente: arquitectos, ingenieros, paisajistas, etcétera. Pero si la noticia es por ejemplo sobre la mega-minería en el páramo de Santurbán, ¿cuál es la relación?

\section{El problema}

Santurbán es un complejo natural de 142.000 hectáreas-ubicado en la cordillera oriental colombiana entre los 2.800 y 4.290 metros sobre el nivel del mar-estratégico como zona de recarga y regulación de agua, donde últimamente resurgió un proyecto que busca explotar durante 23 años las 9 millones de onzas en concentrados de pirita y cobre que se hallan en el lugar, causando gran deterioro ambiental ("Multinacional alista solicitud", 2017). Y se dice resurgió porque, como ocurre con tantos otros desmanes contra la naturaleza, el asunto es de vieja data: "En los páramos de Santurbán y Miraflores sigue explotación minera" (2017), dice el mismo medio cuatro meses antes, y "Pese a fallo de la Corte, sigue la minería en 20 páramos" (2017) unos días después. Y aparecen también las medidas de choque: "Páramo de
Santurbán es declarado parque natural del país" (2013) y "Para conservarlos, veinte páramos del país ya están delimitados" (2016).

Pero las contradicciones no se hacen esperar: si son 36 los ecosistemas definidos como páramos en el país, como bien reza esa última nota, y se delimitaron veinte de ellos-el55,5\%-, ¿cómo se puede denunciar casi dos meses después que "A un año de la decisión, el 70 \% de área de páramos no está delimitada y continúa explotación", si porcentualmente sólo faltaba por delimitar el $44,5 \%$, o sea, dieciséis páramos? ¿Y cómo así que "A un año de la decisión", si la noticia de la delimitación es del 23 de diciembre del 2016 y la de la explotación es del 20 de febrero del 2017, o sea casi dos meses después? ¿Y cuántos páramos son: treinta y seis, como se lee en la noticia, o treinta cuatro, como dice el ministro del Medio Ambiente ("Declaratoria en Santurbán", 2013)? ¿Y sí se ha delimitado alguno? ¿Y hay fallo de la corte? Y si hay fallo, ¿hay penas? Y si hay penas, ¿se cumplieron? Y si no se cumplieron, ¿por qué?

Las preguntas son incontables $\mathrm{y}$, sin embargo, parece que el proyecto sigue en pie, así el "Banco Mundial retira inversión" (2016). Socios, para un proyecto de esas magnitudes $-y$ con esa posibilidad de retorno de inversión, como hablan los economistas-, siempre habrá. Es como si una vez identificado el potencial-hace veinte años, cuando recién llegó Greystar a empezar lo que se convertiría en uno de los proyectos mineros de mayor envergadura de América Latina -, no hubiera manera de detener la acción: denunciaron las comunidades ("Santandereanos marchan", 2011), la prensa alternativa (Duque, 2011; Fierro, 2011), las universidades (Herrera, 2010), los artistas (Observatorio de conflictos mineros de América Latina, 2011), la misma 
Asociación Colombiana de Minería (S.F.), pero nada sirvió: "Solicitan licencia ambiental para explotación minera cerca de Santurbán" (2017), dice la última nota al respecto.

\section{¿A quién culpar?}

Nada se pudo hacer para evitar la explotación y posterior deterioro ambiental del páramo porque, aunque la solución sería “dejar esos recursos enterrados y no explotados", como escribe un columnista citando a Nicholas Stern (Maya, 2013), pareciera que-aún si el medio ambiente está de por medio-ante la opción de lucro no hay como interponerse. Si hoy es en el Páramo de Santurbán, donde intereses particulares se quieren hacer a la riqueza de la montaña, ayer fue en el Chocó cuando se contaminaron las fuentes hídricas durante hace años por el vertimiento de toneladas de químicos, deforestando el bosque húmedo y extinguiendo la fauna nativa ("Policía denunció daño", 2014). Y anteayer fue cuando las operaciones extractivas de madera en comunidades negras del bajo Atrato dejaron graves daños ambientales irreparables, valiéndose y financiándose de estructuras paramilitares (Comisión Intereclesial de Justicia y Paz, 2012). Y así sucesivamente.

Mientras exista un mercado, habrá quien lo explote, diría un capitalista convencido. $\mathrm{O}$, más sencillamente, "Hay una oferta para cada demanda", como sentenció Florence Scovel Shinn hace casi 100 años. Esa teleología justifica la explotación de cualquier recurso natural a costa de lo que haya de por medio. El problema es que todos perdemos, empezando por Santurbán que, de seguir la tendencia de deterioro ambiental actual, se prevé que desaparezca con el $75 \%$ de los páramos y casi la totalidad de los nevados y glaciares de Colombia (Estupiñan, 2015). La pregunta es: ¿todos somos culpables también: ancianos y jóvenes, hombres y mujeres, ricos y pobres?

La primera tentación es decir que no puede ser igualmente culpable, por ejemplo, un hombre de 70 años que manejó durante toda su vida un Rolls Royce Phantom-uno de los modelos más elegantes $\mathrm{y}$, paradójicamente, uno de los más contaminantes (American Council for an
Energy-Efficient Economy, 2014) - , un hombre que conoció los cinco continentes - al transporte aéreo se le atribuye hasta el 9\% del cambio climático (David Suzuki Foundation, 2017) - , y que se alimentó varias veces al día con una dieta a base de cárnicos y lácteos - si el mundo optara comer sólo vegetales y frutas se reducirían las emisiones de la industria alimentaria hasta en un $63 \%$ ("Eat less meat", 2016) -; no puede ser igualmente culpable él que un niño pobre que acabe de nacer en Vetas, el pueblo en la falda de Santurbán que se va a quedar sin actividad económica por cuenta de la explotación minera de alto impacto del páramo... Y no es comparable la emisión colombiana de gases de efecto invernadero a la atmósfera-0,2\% según datos de Greenpeace (2009) - , que la del segundo contaminador del mundo-13,87\%que se retiró recientemente del primer acuerdo ambiental con la participación de 191 países y dejó al mundo en ascuas (Liptak y Acosta, 2017).

No es comparable, en efecto, pero la reparación no puede darse diferencialmente, de manera que la ayuda para revertir el daño esté en relación directa con el aporte a la contaminación (Mathiesen, 2013). Mejor empezar desde cero y decir que la culpa es de todos: de las mineras, de las constructoras, de las madereras, de los grupos al margen de la ley, de la industria química, de las multinacionales, de los políticos y del niño pobre de Vetas. La culpa es hasta de Donald Trump, quien ha negado la emergencia ambiental mundial "más de 115 veces" (Matthews, 2017). No importan las razones, por muy loables que parezcan: para tener ciudades hermosas con oferta suficiente de vivienda para turistas y habitantes; para brindar miles de empleos directos e indirectos; para financiar la lucha armada; para extraer la riqueza de la tierra que de lo contrario se perdería; o para "convertir a América en lo que fue antes", como rezó el eslogan de campaña del presidente de Estados Unidos. En el estado en el que se encuentra actualmente la cuestión, cualquier detrimento a la naturaleza parece escandaloso por muy meritoria que sea la causa. Hace rato se hizo evidente que el esquema capitalista tradicional que considera a la biósfera "como un reservorio inagotable de recursos" no es sostenible en el 
tiempo, que los recursos no son renovables y que de seguir explotándolos indiscriminadamente éstos se acabarán más temprano que tarde (Castro, 2000, p. 47).

\section{La perspectiva geológica}

Hay quienes dicen, sin embargo, que no estamos ante una catástrofe ecológica. Pero no en el sentido del presidente de Estados Unidos cuando dice que "el concepto de calentamiento global fue creado por y para China con el fin de que la industria manufacturera estadounidense no sea competitiva" (Trump, 2012). O en el sentido de los fanáticos religiosos como Hal Lindsay cuando dice que el cambio climático "se está usando para consolidar los gobiernos del mundo en una coalición que algún día puede facilitar la subida del Anticristo" (Vox, 2017). Hay quienes dicen que no estamos ante una emergencia ambiental, porque desde la perspectiva geológica, el calentamiento global presente es un ciclo normal del planeta y no algo que amenace la vida en el planeta.

La explicación, abreviándola al máximo, es que el $\mathrm{CO} 2$ es un gas que siempre ha existido en la tierra-incluso en concentraciones mayores a las que se registran en la actualidad en la atmósfera-, y la actividad volcánica-que se encuentra hoy también en uno de los puntos más altos de la historia - no es un factor determinante para preocuparse, si se tiene en cuenta que los gases tóxicos emitidos por las erupciones de volcanes como el Krakatoa en 1883, el Katmai en 1912 y el Hekla en 1947, son superiores a la cantidad de gases contaminantes producidos por la actividad humana durante toda su existencia (Ruiz, S.F.). Según los expertos, el calentamiento global actual se debe a que el planeta está saliendo de una era de hielo, y aunque la participación de los gases de efecto invernadero producidos por los seres humanos puede haber contribuido en menor medida, no se trata de ninguna manera de su fuente de origen.

La pregunta que surge en ese escenario es cuándo se acabará dicho ciclo de calentamiento. El problema de la respuesta es que al ser humano le cuesta mucho entender procesos que superen su estadía en la tierra, o sea, unos $70 \mathrm{u}$ 80 años - 100 en el mejor de los casos - , y la era de hielo de la que hablan los paleontólogos data aproximadamente del año 1.300 D.C., es decir, del medioevo, hace más de 700 años. Por eso no sirve la analogía del cuento infantil al que acuden con frecuencia los opositores a esta teoría para advertir del riesgo al que está expuesta la humanidad por el deterioro del ambiente: el de la rana en el estanque que se va acostumbrando al aumento de la temperatura del agua hasta morir sin darse cuenta, como se sugiere en Una verdad incómoda (Bender y Guggenheim, 2006), el documental sobre el cambio climático protagonizado por $\mathrm{Al}$ Gore$^{1}$. La tierra sí se está calentando, dicen los que apoyan la teoría geológica, pero por un aumento consistente de las emisiones solares en el último siglo, las que se espera que disminuyan por sí mismas para el año 2030, cuando se produzca de nuevo un descenso de la temperatura (Simanauskas, 2008). Pero éste enfriamiento no será consecuencia de las aparentes medidas de contingencia que toman los hombres-comprar bombillos ahorradores de energía, separar la basuras, montar en bus en lugar de utilizar el carro particular, etcétera-. No es que los seres humanos vayan a solucionar el cambio climático.

\section{El antropoceno}

Por otro lado están los que dicen que el mundo sí está ante una tragedia ecológica. Pero esos son demasiados para ser nombrados. Mejor buscar un término que pueda englobarlos a todos.

1 Según lo demostró el fisiólogo alemán Friedrich Leopold Goltz en 1869, la rana se quedará dentro del estanque, esperando cómodamente la muerte mientras la temperatura aumenta, sólo si se le ha sacado el cerebro con anterioridad. Si la rana tiene su cerebro intacto, usualmente salta del agua despavorida cuando el agua llega a $25^{\circ} \mathrm{C}$ (Fallows, 2009). La paradoja es que si se quiere que el Síndrome de la Rana Hirviendo sirva de ejemplo, habría que pensar en la humanidad como sin cerebro. De otra manera, biológicamente, es imposible. 
Tal como lo reportó la prensa ambientalista mundial (Fulton, 2016), el año 11.700 del Holoceno llegó a su fin y hoy estamos en lo que se llama ahora el antropoceno: una nueva época geológica causada, no por los miles o millones de años de los procesos naturales evolutivos, sino por la actividad humana, la cual cambió dramáticamente la trayectoria de los sistemas globales dejando registro geográfico (Waters et al., 2016).

El informe oficial del XXXV Congreso Geológico Internacional explicó la decisión con 9 situaciones, considerando su impacto estratigráfico en el planeta y al hecho de que son modificaciones irreversibles en la mayoría de los casos: 1) la aceleración de la erosión y la sedimentación; 2) la perturbación de los ciclos de carbono, nitrógeno, fósforo y otros elementos; 3) los cambios al clima global y al nivel de los mares; 4) las cantidades sin precedente de especies invasoras a lo largo y ancho de la tierra; 5) los radionúclidos artificiales en el ambiente; 6) los cambios a los patrones de los isótopos de carbón y nitrógeno; 7) las partículas de plástico, aluminio y concreto en el suelo; 8) las partículas de ceniza en el aire; y 9) los restos biológicos fosilizables (Anthropocene Working Group AWG, 2016).

No obstante, aún existe un fuerte debate sobre cuándo empezó oficialmente el antropoceno. Se estima que la fecha son los años posteriores al fin de la segunda guerra mundial-1945-, debido a la rápida industrialización, las pruebas nucleares, la agricultura extensiva y la proliferación del plástico (Steffen et al., 2011). Pero aparte de la complejidad disciplinar que implica sustentarlo, además de los alcances que esto redunda en otras ciencias-incluyendo la arquitectura y la planeación urbana-, ¿qué significa que el Homo Sapiens tenga su propia época geológica? Y además, ¿encontrando ese momento de quiebre anterior, se puede pensar en regresar y retomar el rumbo: no regresar en el sentido de viajar en el tiempo hacia el pasado y comenzar de nuevo, naturalmente, pero sí regresar en el sentido de volver a otras maneras de convivir con la naturaleza y corregir todo aquello que trajo a los humanos hasta esta encrucijada?

\section{La madre naturaleza}

Don Adonaí Guerrero, un hombre de cuarenta y ocho años que trabaja desde los veintitrés como minero en el pueblo de Vetas en el páramo de Santurbán, dice que sí: "Llevamos cuatrocientos sesenta años desarrollando actividades mineras [aquí], pero estas son de carácter tradicional y en muy poca extensión" ("El fracaso de Santurbán", 2016).

Afirmaciones como ésta-así como la decisión de marcar el comienzo del antropoceno en los años 50 del siglo XX y no antes, mucho antes-sugieren que en el pasado fue posible sacar de la tierra los recursos necesarios para la supervivencia de la especie humana sin destruir el aparente equilibrio natural. La pregunta que surge es de nuevo cuándo: ¿hace 70.000 años, cuando el Homo Sapiens se separó de sus homínidos relacionados por la llamada revolución cognitiva y empezó la civilización humana (Dawson, 2016); o hace 8.000 años, cuando el hombre se volvió sedentario y domesticó animales y empezó la revolución agraria (Ruddiman, 2003)? ¿O hace 500 años, en la fecha del descubrimiento de América, cuando se dio el intercambio de especies entre el viejo y el nuevo mundo; o hace menos de 200 años, hacia la revolución industrial, cuando las emisiones de carbón se dispararon por cuenta de los nuevos inventos (Crutzen y Stoermer, 2000)?

Hay numerosas estudios que consideran el Conocimiento Ecológico Tradicional (TEK, Traditional Environmental Knoledge) como una alternativa viable para pensar el futuro de la humanidad, involucrando aquellos saberes dejados de lado por no considerarse científicos, para así modelar conjuntamente sistemas de desarrollo futuros, más conscientes de los daños que se pueden ocasionar al ambiente: porque el conocimiento de las culturas aborígenes es holístico y no lineal (Freeman, 1992); porque la sabiduría ancestral está oculta en idiomas y dialectos que desaparecen rápidamente (Kothari, 2007); por las versátiles técnicas de gestión de los recursos que muestran los pueblos indígenas (Berkes, Colding y Folke, 2000); o para recuperar las nociones vernácula de propiedad común 
y gestión cooperativa de la tierra (Doubleday, 1993), entre muchas otras.

Sin embargo, a pesar de todas las aparentes ventajas de esa comunión, no deja de haber problemas. Para Sandom, Faurby, Sandel y Svenning (2014) la condición de nativos no los hace necesariamente mejores, pues la evidencia científica indica que fue la llegada de los hombres a todos los rincones de la tierra la que acabó con los mamíferos mayores a $10 \mathrm{~kg}$ en todo el mundo, lo que a juicio de otros académicos constituye la primera catástrofe ecológica de la que se tenga noticia:

"No les crea a esos amantes de los árboles que claman que nuestros ancestros vivieron en armonía con la naturaleza. Mucho antes de la revolución industrial, entre todos los organismos el Homo Sapiens tenía el record de llevar más especies de animales y plantas a su extinción. Tenemos la dudosa distinción de ser la especie más mortífera en los anales de la biología. Tal vez si la gente fuera consciente de las extinciones de la Primera y Segunda Olas, sería menos indulgente con la Tercera Ola de la que hacen parte. Si supiéramos cuántas especies ya hemos erradicado, estaríamos más motivados a proteger aquellas que aún sobreviven" (Harari, 2015).

Por su parte Briggs (2005), observa que el asunto "más espinoso" es que el TEK está enraizado en su contexto local, mientras que el conocimiento científico occidental prospera en la formulación abstracta de problemas. Por esta razón el conocimiento indígena se asume en occidente como trozos pequeños de información que se pueden hacer coincidir en los compartimientos definidos en los paradigmas existentes, como si fueran soluciones rápidas, descontextualizadas, lo que despoja la sabiduría tradicional de su relevancia, su aplicabilidad, e incluso su poder. McGregor (2004) añade que es precisamente esa contextualización, ese arraigo del hombre-no a la tierra, en general, sino a su tierra-, lo que deriva en que haya culturas tradicionales donde el carácter simbólico más importante sea una montaña, mientras para otras puede ser un animal o un árbol o un río, y que no para todas sea siempre igual.
Claramente esto va en contravía del pensamiento occidental, que anhela lo global (Mattelart, 2000). Pero tal vez en un intento de unir esos dos bandos en procura de proteger el ambiente entre todos, en un reciente comercial se vuelve a la tierra como lo humano común, usando quizás premeditadamente el animismo que tanto caracteriza al pensamiento tradicional. La naturaleza nos habla:

"Algunos me llaman 'naturaleza'; otros me llaman 'madre naturaleza'. He estado aquí más de cuatro billones de años (sic); veintidós mil quinientas veces más que tú. Yo realmente no necesito a la gente, pero la gente necesita de mí. Sí: tu futuro depende de mí. Cuando yo prospero, tú prosperas. Cuando yo vacilo, tú vacilas... O aún peor. Pero he estado aquí por miles deaños; he alimentado especies más grandes que la tuya y he matado de hambre ha especies más grandes que la tuya. Mis océanos, mi suelo, mis torrentes de agua, mis bosques: todos ellos pueden acompañarte o dejarte. Cómo escojas vivir cada día, sea considerándome o ignorándome, realmente a mí no me importa. De un modo $u$ otro, tus acciones determinarán tu destino, no el mío. Yo soy la naturaleza. Yo seguiré adelante. Yo estoy preparada para evolucionar. ¿Lo estás tú?" (Conservación internacional, 2014).

La voz que le da vida a la naturaleza en el video es la de Julia Roberts, la actriz de Hollywood, y el éxito fue tal que la demanda creció de manera virulenta hasta el punto que, donde antes había 1 comercial, ahora hay 12: Harrison Ford es el océano, Liam Neeson es el hielo, Joan Chen es el cielo, Lee Pace es la montaña, Reese Witherspoon es el hogar, Kevin Spacey es el bosque húmedo, Edward Norton es el suelo, Penélope Cruz es el agua, Robert Redford es la secuoya, Ian Sommerhalder es la barrera coralina y Lupita Nyong' o es la flor. Y hay más en camino. No hace falta decir que la estrategia se puede extender indefinidamente.

He ahí la gran diferencia entre el conocimiento tradicional y la ciencia occidental, tal como lo identifica Mazzocchi (2006): “Las sociedades tradicionales están más interesadas en preservar la estabilidad y la integridad de sus propias sociedades, culturas y ambientes, que en 
maximizar la producción". Por eso el comercial a sus ojos no se ve como un acto genuino por el ambiente, sino no por el capitalismo. Porque en la sabiduría ancestral proteger la naturaleza no quiere decir introducir la problemática ambiental dentro del sistema del capital, para entonces proteger la naturaleza a partir de la venta de camisetas y pulseras y afiches y grabaciones, que significan además mayor explotación de los recursos de la naturaleza. Proteger la naturaleza puede ser más bien no hacer: no cortar árboles para hacer papel, no barrer el fondo marino en busca de camarones, no verter las aguas negras a las fuentes hídricas, etc. Pero no hacer va en contra de la filosofía del capital, que es hacer: producir y consumir y generar plusvalías y producir riqueza.

Por esa razón tantos dudan de que el propósito se pueda cumplir. En el caso de Gran Bretaña, por ejemplo-que es un país $100 \%$ capitalista-, los analistas no se pueden explicar cómo van a hacer para bajar las emisiones de gases de efecto invernadero hasta en un $80 \%$ para el año 2050, como lo indican las leyes de cambio climático emitidas en el 2008, sin que colapse la economía por los 18 mil millones de libras esterlinas anuales que costará hacerlo (Booker, 2010). Porque se asume que los habitantes de Londres y de Manchester y de Liverpool no dejarán de hacer nada que comprometa su estilo de vida; se da por hecho que la vida seguirá igual, o incluso mejor; que continuarán siendo una de las grandes potencias mundiales: "El problema es que la mayoría de nosotros no quiere ser verde si eso significa perder de un fin de semana en Barcelona, calentar una casa más amplia o manejar un carro más grande" (Kasterine, 2009). Si sin renunciar a aquellos placeres la tierra se salva con las medidas programadas, bien. Pero si es porque los aviones no queman combustibles fósiles o porque la calefacción ahorra energía o porque el carro emite menos gases, eso al comprador promedio no le interesa. Lo importante es que no se detenga el consumo.

\section{Capitalismo vs Naturaleza}

En ese sentido dice Zhang (2013, p.71), que el capitalismo es "la raíz de la crisis ecológica" y añade-como pensando en Santurbán-que es el proceso de globalización, sumado al capitalismo y a la conquista de la naturaleza por los medios de producción, lo que cada día más "asola al mundo". O sea que el enfrentamiento es entre la ciudad y la naturaleza, porque es la ciudad-en la dinámica del capitalismo-la que conquista y destruye la naturaleza: es la ciudad la que quiere la pirita y el cobre del Páramo de Santurbán, la que demanda viviendas en la Sierra Nevada de Santa Marta, la que necesita la madera que crece en los árboles que protegen la cuenca del río Atrato, la que requiere los químicos que se extraen de las plantas del bosque húmedo del Chocó... Si la naturaleza habla, las ciudades gritan.

Por eso cuando se trata de proteger el ambiente se piensa necesariamente en ciudades sostenibles y ecourbanismo (Sarkar, 2000). Porque el desarrollo sólo se concibe en un esquema urbano. $\mathrm{Y}$ mientras no encontremos maneras de que las ciudades - "epicentro del desarrollo económico del país", como se les llamó recientemente (España, 2017)-cuiden la naturaleza, el panorama es muy desalentador. Así lo prevé el último informe del Instituto de Hidrología, Meteorología y Estudios Ambientales (IDEAM) ("Ciudades y cambio climático", 2017), donde se sentencia que 15 de las 32 capitales de Colombia están en alto riesgo ambiental ante los escenarios que se proyectan para el 2040.

Es lógico que al final la culpa caiga sobre las ciudades: en el reporte del Programa de Naciones Unidas para el Ambiente (UNEP), cuya nota periodística lleva el alarmante título de "Voraz consumo de recursos naturales por parte de la humanidad es insostenible" (UNEP, 2011), se explica que ya se acaban las reservas de petróleo, oro y cobre-cobre del que hay por toneladas en Santurbán - y en el Reporte Global sobre los Asentamientos Humanos, también de Naciones Unidas, se relaciona directamente este consumo voraz con el desarrollo urbano mundial, diciendo incluso que los planificadores urbanos deben culparse en gran parte por el estado actual del ambiente (Garau, 2009, p. 32).

Según la Organización de Naciones UnidasONU-, en las ciudades reside el $54,5 \%$ de la 
población mundial y se espera que el en 2030 ese porcentaje se eleve hasta el 60\% (ONU, 2017). Pero esos números son sobre los centros propiamente dichos: si se tiene en cuenta la zona metropolitana de las ciudades, o sea, los habitantes de los sectores suburbanos, periféricos y rurales, entonces es el $97,6 \%$ de la población mundial el que vive en algún modo de urbanización: 7.389 millones de personas según el reloj poblacional en tiempo real (Worldometers, 2017). Lógicamente el comportamiento de esa población con respecto al consumo de recursos y al manejo de residuos será de primera importancia para la buena salud del ambiente en el futuro.

Es claro que cuando se habla de protección ambiental lo primero que se piensa no es en Nueva York o en Beijing, sino en la naturaleza que nos habla en el comercial, sin seres humanos, o con muy pocos seres humanos. Porque así como no se muestran en el video grandes asentamientos urbanos, tampoco se muestran pueblos ni caseríos, y ni siquiera campos de cultivos de arroz o planicies hermosas para criar ganado, donde se sugiera alguna presencia homínida. Por el contrario, se ven sólo picos inalcanzables y ríos torrentosos y desiertos inclementes y profundidades abisales sin ningún barco pesquero a la vista. Esa naturaleza salvaje, según los cálculos de la misma Conservación Internacional (Mittermeier, Mittermeier, RoblesGil y Pilgrim, 2003), ya corresponde a un poco menos de la mitad de la superficie de la tierraal $46 \%$-y está habitada por sólo el $2,4 \%$ de la población mundial, o sea, 189 millones de personas.

El problema es que ese $46 \%$ de superficie con bajísima población humana ya no es necesariamente naturaleza salvaje, como sugeriría el sentido común. Si se observa con detenimiento, salvo por unas algas y un cardumen de pececillos justo antes del final, el comercial tampoco muestra animales de ningún tamaño, ni grandes ni chicos, ni mamíferos ni reptiles ni insectos. Se muestra una naturaleza deshabitada en todo el sentido de la palabra, no como la concibe el pensamiento occidental o ancestrales, sino llena de vida microscópica en potencia, antes de la emergencia del proceso que nos trajo a lo largo de millones de años.
La pregunta queda flotando en el aire:

- ¿Cómo abogar por el cuidado de una naturaleza de la que no tenemos conciencia, pues no la conocemos sin nosotros (sin nuestra huella, que son las ciudades?

La respuesta puede tener ribetes filosóficos, pero por ahora urge una réplica inmediata:

- Porque es lo único que nos queda.

\section{Referencias bibliográficas}

American Council for an Energy-Efficient Economy - ACEEE. (2014). Least and Most Polluting Cars. Recuperado de https://goo. gl/OKnwBM

Anthropocene Working Group - AWG (2016). Media note. Agosto 29. Recuperado de Universidad de Leicester. https://goo.gl/ AWndVB

Asociación Colombiana de Minería (S.F.). ¿Vuelve la minería ilegal al Páramo de Santurbán? Recuperado de https://goo.gl/ wp93KF

Banco Mundial retira inversión de proyecto minero en Santurbán (2016, Diciembre 20), El Tiempo. Recuperado de https://goo.gl/ bs2VFY

Bender, L. (Productor) \& Guggenheim, D. (Director) (2006). Una verdad incómoda (Motion Picture). Estados Unidos: Paramount Classics.

Berkes, F., Colding, J. \& Folke, C. (2000). Rediscovery of traditional ecological knowledge as adaptive management. Ecological Applications, 10(5), 1251-1262.

Booker, C. (2010, Mayo 29). The woolly world of Chris Huhne. The Telegraph. Recuperado de https://goo.gl/6Lpt4u

Briggs, J. (2005) The use of indigenous knowledge in development: problems and challenges. Progress in Development Studies 5(2):99-114. 
Castro, G. (2000). La crisis ambiental y las tareas de la historia en América Latina. Papeles de Población 6(24): 37-60.

Ciudades y cambio climático (2017, Junio 23), El Tiempo. Recuperado de https://goo.gl/ QXM2Gm

Comisión Intereclesial de Justicia y Paz. (2012). Maderas del Darién Pizano S.A causa daño ambiental en Bajo Atrato. Recuperado de https://goo.gl/3GIK5m

Conservación internacional (2014). Nature is speaking. Recuperado de https://goo.gl/ OnDhBc

Crutzen, P.J. \& Stoermer, E.F. (2000). The 'Anthropocene'. Global Change Newsletter 41, 17-18.

David Suzuki Foundation. (2017). Air travel and climate change. Recuperado de https://goo. $\mathrm{gl} / \mathrm{zaaWI7}$

Dawson, A. (2016). Extinction: A Radical History. New York, NY: OR Books.

Declaratoria en Santurbán es parte de una política de Estado (2013, Enero 9), El Tiempo. Recuperado de https://goo.gl/c8NWSC

Doubleday, N. (1993). Finding common ground: natural law and collective wisdom (pp. 41-53). Inglis, J. (ed.). Traditional ecological knowledge: concepts and cases. Ottawa, Canada: International Development Research Centre.

Duque, M.V. (2011, Enero 24). Minería en el Páramo de Santurbán: ¡No viable! Razón Pública. Recuperado de https://goo.gl/ qfDKZk

Eat less meat to avoid dangerous global warming, scientists say (2016, Marzo 21). The Guardian. Recuperado de https://goo. $\mathrm{gl} / \mathrm{sFKqpl}$

El fracaso de Santurbán (2016, Abril 9), Semana. Recuperado de https://goo.gl/F3Dfn5
En los páramos de Santurbán y Miraflores sigue explotación minera (2017, Febrero 9), El Tiempo. Recuperado de https://goo.gl/ a8xu8d

España, C. (2017, Septiembre 19). Ciudades colombianas: epicentro del desarrollo económico del país. Portafolio. Recuperado de https://goo.gl/gDefhA

Estupiñan, L.F. (2015, Noviembre 3). La mayoría de páramos en Colombia está en riesgo de desaparecer, según estudio. Recuperado de https://goo.gl/6fHl43

Fallows, J. (2009, Julio 21). Guest-post wisdom on frogs. The Atlantic. Recuperado de https://goo.gl/ijvkRo

Fierro, J. (2011, Marzo 28). Minería en los páramos: el agua vale más que el oro. Razón Pública. Recuperado de https://goo. $\mathrm{gl} / 4 \mathrm{P} 4 \mathrm{dxd}$

Freeman, M. (1992). The nature and utility of traditional ecological knowledge. Northern Perspectives, 20(1). Recuperado de https:// goo.gl/S6Dz4m

Fulton, D. (2016). It's official: the Anthropocene epoch is here. Ecowatch. Recuperado de https://goo.gl/Bzh963

Fundación ProAves de Colombia (2015). ¡Alerta Ambiental! Máquinas excavadoras destruyen la Sierra Nevada de Santa Marta. Recuperado de https://goo.gl/PGE25m

Garau, P. (2009). Revisiting Urban Planning in Developed Countries. Report on asdfasdf Recuperado de https://goo.gl/oj6mQc

Greenpeace. (2009). Páramos en peligro. Recuperado de https://goo.gl/WyeEZL

Harari, Y.N. (2015). Sapiens: a brief history of humankind. New York, NY: Harper Collins.

Herrera, E. (2010). Páramo de Santurbán, de la minería 'artesanal' a la explotación 
multinacional. Recuperado de https://goo. $\mathrm{gl} / \mathrm{XIGhBW}$

Kasterine, A. (2009, Marzo 31). Carbon labels present taxing problem. BBC News. Science and environment. Recuperado de http:// news.bbc.co.uk/2/hi/7974972.stm

Kothari, A. (2007). Traditional knowledge and sustainable development. Winnipeg, Canada: International Institute for Sustainable Development.

Liptak, K. \& Acosta, J. (2017, Junio 2). Trump on Paris accord: "We're getting out". Recuperado de https://goo.gl/UvsfqY

Mathiesen, K. (2013, Noviembre 20). Climate talks: Should rich countries pay for damage caused by global warming? The Guardian. Recuperado de https://goo.gl/pzCVje

Mattelart, A. (2000). Historia de la utopía planetaria: de la ciudad profética a la ciudad global. Barcelona: Paidós Ibérica.

Matthews, D. (2017). Donald Trump has tweeted climate change skepticism 115 times. Here's all of it. Recuperado de https://goo. $\mathrm{gl} / \mathrm{iFtE32}$

Maya, G. (2013, enero 27). Santurbán: a merced de la gran minería. Recuperado de https:// goo.gl/rkMhLu

Mazzocchi, F. (2006). Western science and traditional knowledge: despite their variations, different forms of knowledge can learn from each other. EMBO Reports, 7(5), 463-466.

McGregor, D. (2004). Traditional ecological knowledge and sustainable development: towards coexistence (pp. 72-91). En Blaser, M., Feit, H. \& McRae, G. (Eds.). In the way of development: indigenous peoples, life projects and globalization. London \& New York: Zed Books.
Mittermeier, R.A., Mittermeier, C.G., Robles-Gil, P. \& Pilgrim, J. (2003). Wilderness: Earth's Last Wild Places. Conservation International: University of Chicago Press.

Multinacional alista solicitud de licencia ambiental para explotación (2017, Junio 2), El Tiempo. Recuperado de https://goo. $\mathrm{gl} / 1 \mathrm{j} 10 \mathrm{Oa}$

Observatorio de conflictos mineros de América Latina - OCMAL. (2011, Febrero 23). Artistas respaldan jornada en defensa del páramo de Santurbán. Recuperado de https://goo.gl/HOks6x

Organización de Naciones Unidas - ONU (2017). The world's cities in 2016. Recuperado de https://goo.gl/pVdtwy

Para conservarlos, veinte páramos del país ya están delimitados (2016, Diciembre 23), El Tiempo. Recuperado de https://goo. $\mathrm{gl} / 9 \mathrm{DKMoG}$

Páramo de Santurbán es declarado parque natural del país (2013, Enero 8), Portafolio. Recuperado de https://goo.gl/eEYvVq

Pese a fallo de la Corte, sigue la minería en 20 páramos (2017, Febrero 20), El Tiempo. Recuperado de https://goo.gl/5t7yT3

Policía denunció daño ambiental en Chocó (2014, Marzo 14), El Universal. Recuperado de https://goo.gl/BtV2tM

Programa de Naciones Unidas para el Ambiente - UNEP (2011). Humanity's voracious consumption of natural resources unsustainable. Recuperado de https://goo. gl/SbwZgg

Ruddiman, William F. (2003). The anthropogenic greenhouse era began thousands of years ago. Climatic Change, 61(3), 261-293.

Ruiz, M. (S.F.). Los disidentes de la teoría sobre el calentamiento global. Recuperado de https://goo.gl/Q93fQd 
Sandom, C., Faurby, S., Sandel, B. \& Svenning, J.C. (2014). Global late quaternary megafauna extinctions linked to humans, not climate change. Procedings of the Royal Society B, 281(1787). http://doi.org/10.1098/ rspb.2013.3254

Santandereanos marchan contra minería en Santurbán. (2011, Febrero 21), El Espectador. Recuperado de https://goo.gl/p6WsPl

Sarkar, S. (2000). ¿Eco-socialism or eco-capitalism?: a critical analysis of humanity's fundamental choices. Telangana, India: Orient Blackswan.

Simanauskas, T. (2008). Calentamiento global: un cambio climático anunciado. Buenos Aires, Argentina: Ediciones Continente.

Solicitan licencia ambiental para explotación minera cerca de Santurbán (2017, Septiembre 7), El Espectador. Recuperado de https://goo.gl/ugCKux

Steffen, W., Persson, Å., Deutsch, L., Zalasiewicz, J., Williams, M., Richardson, K., ... Svedin, U. (2011). The Anthropocene: From Global Change to Planetary Stewardship. Ambio,
40(7), 739-761. http://doi.org/10.1007/ s13280-011-0185-x

Trump, D. (2012, Noviembre 6). The concept of global warming was created by and for the Chinese in order to make U.S. manufacturing non-competitive [tweet]. Recuperado de https://goo.gl/iqdPKL

Vox, L. (2017, Junio 2). Why don't Christian conservatives worry about climate change? God. The Washington Post. Recuperado de https://goo.gl/e8xEe1

Waters, C.N., Zalasiewicz, J., Summerhayes, C., Barnosky, A.D., Poirier, C., Gałuszka, A., ... Ellis, E.C. (2016, Enero 8). The Anthropocene is functionally and stratigraphically distinct from the Holocene. Science 351(6269). http://doi.org/10.1126/science.aad2622

Wordometers (2017). Current world population. Recuperado de https://goo.gl/7JZSE

Zhang, Y. (2013). Capitalism and ecological crisis. Journal of Sustainable Society, 2(3), 6973. 